\title{
Development and Evaluation of Curcumin Transferosomal Hydrogel: - A Promising Treatment for Psoriasis
}

\author{
Yogesh Kumar Baliarsingh ${ }^{1 *}$, A.K. Singhai ${ }^{1}$, Nisha Thakre ${ }^{1}$, Subhendu S. Mishra ${ }^{2}$ \\ ${ }^{1}$ Lakshmi Narain College of Pharmacy, Bhopal, M.P, India \\ ${ }^{2}$ Kalinga Biomedical Research Institute \& Training Establishment, Sambalpur, Odisha, India \\ *Corresponding Author's E mail: rahulbaliarsingh78@gmail.com
}

Received 23 July 2020; Revised 28 July 2020; Accepted 12 Aug. 2020, Available online 10 October 2020

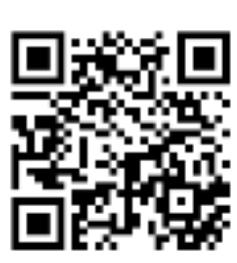

Cite this article as: Baliarsingh YK, Singhai AK, Thakre N, Mishra SS. Development and Evaluation of curcumin transferosomal Hydrogel: - A promising treatment for Psoriasis 2020; 9(4): 98-107.

https://dx.doi.org/10.38164/AJPER/9.3.2020.96-106.
\end{abstract}

\begin{abstract}
The aim of this work to develop curcumin transferosomal gel for the better treatment of Psoriasis through skin. The transferosome prepared by Modified Hand Shaking Method. In this method Soya-phosphatidylcholine, Span 80 and Salicylic acid were dissolved alcohol. Then solution was put in a round bottom flask. These were then dissolved by shaking. The mixture is subjected to evaporation to remove the organic solvent using temperature above the transition temperature of lipid by hand shaking. The characterization of trasferosome were further evaluated for vesicle size, drug entrapment efficiency, Shape and surface characterization by Microscopic observation. The vesicle size of all transfersomes varied between 178.89 and $245.65 \mathrm{~nm}$ whereas entrapment efficiency was found between 61.15 to $75.65 \%$. the characterization of transferosomal gel were further evaluated for $\mathrm{pH}$, viscosity, spreadability, drug content and invitro release study. The Viscosity of optimized formulation was found to be was in the range of $2361 \pm 0.046-2772 \pm 0.058$ cps. In-vitro diffusion study of the transfersomes gel (F1-F6) was performed using modified Franz diffusion cell with dialysis membrane in phosphate buffer $\mathrm{pH} 7.4$ for a period of 12 hours. In vitro release of the drug was found in the rage of 80.269-89.230.
\end{abstract}

Keywords: Curcumin, Transferosome, Soya-phosphatidylcholine, invitro release study.

\section{INTRODUCTION}

Transferosomes are the optimized ultra-deformable lipid supramolecular aggregates able to penetrate mammalian skin. The edge activator is incorporated into the layers such as span 80, tween 80. They consist of at least inner aqueous phase surrounded by lipid bilayer. ${ }^{1}$ A Transfersome carrier is an artificial vesicle designed to exhibit the characteristics of a cell vesicle or a cell engaged in exocytosis, and thus suitable for controlled and, potentially, targeted drug delivery. ${ }^{2}$ A Transfersome carrier is an artificial vesicle designed to exhibit the 
characteristics of a cell vesicle or a cell engaged in exocytosis, and thus suitable for controlled and, potentially, targeted drug delivery. ${ }^{3}$

Hydrogels are water swollen polymer matrices, with a tendency to imbibe water when placed in aqueous environment. ${ }^{4}$ This ability to swell, under biological conditions, makes it an ideal material for use in drug delivery and immobilization of proteins, peptides, and other biological compounds. Due to their high water content, these gels resemble natural living tissue more than any other type of synthetic biomaterial. These networks, have a three dimensional structure, cross-linked together either physically (entanglements, crystallites), or chemically (tie-points, junctions) ${ }^{5,6}$

Curcumin is chemically (1E, 6E)-1, 7-bis (4-hydroxy-3- methoxyphenyl) hepta-1, 6-diene-3, 5Dione. Curcumin is used for the treatment of anti-cancer, anti-oxidant, antiinflammatory, hyperlipidemic, antibacterial, wound healing and hepatoprotective activities. Apart from its pharmacological actions, it has also been investigated as photostabilizing agent to protect photolabile drugs in solution, topical preparations and soft gelatin capsules. Despite the presence of large number of pharmacological actions, the therapeutic efficacy of curcumin is limited due to its poor oral bioavailability. The poor oral bioavailability of curcumin has been attributed to its poor aqueous solubility as its partition coefficient 3.2 and extensive first pass metabolism. ${ }^{7}$

The objective of the study to develop hydrogel of curcumin in the form of transfersome to get enhanced bioavailability and long term treatment of psoriasis. The safety and efficacy of a novel occlusive hydrogel dressing was assessed in an open-label, bilaterally controlled study that compared the use of hydrogel dressing alone vs hydrogel dressing in conjunction with topical medications (hydrocortisone cream; tacrolimus ointment; triamcinolone cream $0.1 \%$; calcipotriene cream $0.05 \%$; halobetasol propionate cream $0.05 \%$ and calcipotriene cream $0.005 \%$ ) in the treatment of plaque psoriasis.

\section{MATERIALS AND METHODS}

\section{Material}

Curcumin was purchased on himedia lab, Mumbai, Soya Lecithin and span 80 purchase from HIMEDIA Lab Mumbai. Carbopol and PEG purchased from Loba Chem. Pvt. Ltd., Mumbai Tween 80 was purchase from HIMEDIA Laboratories Pvt. Ltd, Mumbai, All other chemicals were locally procured and solvents of merck grade were used. 


\section{Method}

\section{Formulation and Development of Curcumin Transferosome}

Soya lecithin and span 80 are taken at different ratios. After that $100 \mathrm{mg}$ drug is added, dissolving in a mixture of chloroform and methanol with ratio $1: 1$. The mixture is evaporated to remove organic solvent above the transition temperature of liquid by hand shaking. A thin film is form which is left over night to ensure complete removal of solvent. A buffer capsule is tore into a 100 $\mathrm{ml}$ of beaker with $10 \mathrm{ml}$ water. Gently shake the solution for 5 minutes. Makeup the volume upto $100 \mathrm{ml}$. The film is hydrated with buffer water in gentle hand shaking for $15 \mathrm{~min}$. The suspension formed is further hydrated at $40^{\circ} \mathrm{C}$ for $40 \mathrm{~min}$. Brownish colored tranferosomal suspension is formed. ${ }^{8,9}$

Table 1: Formulation code and variable used in preparation of transferosome.

\begin{tabular}{cccc}
\hline S.NO & $\begin{array}{c}\text { FORMULATION } \\
\text { CODE }\end{array}$ & SL:S & DRUG(MG) \\
\hline 1 & SP1 & $95: 05$ & 100 \\
2 & SP2 & $90: 10$ & 100 \\
3 & SP3 & $85: 15$ & 100 \\
4 & SP4 & $80: 20$ & 100 \\
5 & TW5 & $80: 20$ & 100 \\
6 & TW6 & $85: 15$ & 100 \\
7 & TW7 & $90: 10$ & 100 \\
8 & TW8 & $95: 05$ & 100 \\
\hline
\end{tabular}

(SP-Span 80, TW-Tween 80, SL-Soya lecithin, S-surfactant)

\section{Characterization of Curcumin-loaded transfersomes:}

\section{Vesicle size distribution and zeta potential ${ }^{10,11}$}

Vesicle size, size distribution and zeta potential were determined by Dynamic Light Scattering system by Malvern Zetasizer.

\section{Vesicle morphology}

The diameter of vesicle in a transferosome can be determined by dynamic light scattering method. The samples are passed through $0.2 \mathrm{~mm}$ diameter of membrane filter. Before the samples are 
prepared by distilled water. Filtered sample is diluted with saline which is also filtered. Then size measurement is done by dynamic scattering method. Although the vesicle of transferosome can be visualized by TEM. Stability of the vesicle determined by size and structure of the vesicle. Mean size of the vesicles is performed by DLS method. System by Malvern Zetasizer.

\section{Entrapment efficiency ${ }^{12}$}

Percentage of drug which are entrapped into a delivery system is known as entrapment efficiency. By the use Mini column centrifugation method the first separation of the unentrapped drug can be determined. After centrifugation, the vesicles were disrupted using $0.1 \%$ Triton $\mathrm{X}-100$ or $50 \% \mathrm{n}$ propanol. The entrapment efficiency is expressed as:

Entrapment efficiency $=($ Amount entrapped $/$ Total amount added $) \times 100$

\section{Surface charge and charge density}

Surface charge and charge density of Transfersomes can be determined using zetasizer.

\section{PREPARATION OF TRANSFERSOME HYDROGELS}

For the preparation of hydrogel, the gel-forming polymer Carbopol 971P (2\%) was dispersed in distilled water, stirred for $10 \mathrm{~min}$ at $1500 \mathrm{rpm}$ and neutralized by triethanolamine until $\mathrm{pH}$ 6.0. Hydrogel were further allowed to equilibrate for 24 hours at room temperature and then used to disperse a freshly prepared tropical hydrogel. Transferosomal dispersion and hydrogel were mixed in a high speed stirrer (Remi, Mumbai, India) at $1000 \mathrm{rpm}$ for the next $5 \mathrm{~min}$. The gel was allowed to stand overnight to remove entrapped air.

Table 2. Formulation of topical hydrogel

\begin{tabular}{|c|c|c|c|c|}
\hline \multirow[t]{2}{*}{ Formulation } & \multicolumn{4}{|c|}{ Topical Hydrogel formulations } \\
\hline & HG1 & HG2 & HG3 & HG4 \\
\hline Transfersome & $\begin{array}{l}\text { Eqv. To } 2 \% \\
\text { of drug }\end{array}$ & $\begin{array}{l}\text { Eqv. To } 2 \% \\
\text { of drug }\end{array}$ & $\begin{array}{l}\text { Eqv. To } 2 \% \\
\text { of drug }\end{array}$ & $\begin{array}{l}\text { Eqv. To } 2 \% \\
\text { of drug }\end{array}$ \\
\hline Carbopol971P & $0.5 \%$ & $1.0 \%$ & $1.5 \%$ & $2.0 \%$ \\
\hline $\begin{array}{l}\text { Propylene } \\
\text { Glycol }\end{array}$ & $10 \%$ & $10 \%$ & $10 \%$ & $10 \%$ \\
\hline Glycerol & $30 \%$ & $30 \%$ & $30 \%$ & $30 \%$ \\
\hline Distilled water & \multicolumn{4}{|c|}{ q.s } \\
\hline
\end{tabular}




\section{CHARACTERIZATIONOF TOPICAL HYDROGEL}

\section{Determination of $\mathrm{pH} \mathbf{1}^{13}$ :}

The value of $\mathrm{pH}$ of topical hydrogels was measured by using digital $\mathrm{pH}$ meter (EI. Alpha $01 \mathrm{pH}$ meter) at the room temperature.

\section{In-vitro drug release of transferosomal hydrogel}

An in-vitro drug release study was performed using modified Franz diffusion cell. Dialysis membrane (Hi Media, Molecular weight 5000 Daltons) was placed between receptor and donor compartments. Transfersomes hydrogel equivalent to $500 \mathrm{mg}$ of curcumin was placed in the donor compartment and the receptor compartment was filled with phosphate buffer, $\mathrm{pH} 7.4$ (24 ml). The diffusion cells were maintained at $37 \pm 0.5^{\circ} \mathrm{C}$ with stirring at $50 \mathrm{rpm}$ throughout the experiment. At different time interval, $5 \mathrm{ml}$ of aliquots were withdrawn from receiver compartment through side tube and analyzed for drug content by UV Visible spectrophotometer $424 \mathrm{~nm}$ (Rizwan et al., 2004). The quantitative analysis of the values obtained in dissolution/release tests is easier when mathematical formulas that express the dissolution results as a function of some of the dosage forms characteristics are used ${ }^{14,15}$.

\section{RESULTS AND DISCUSSION}

\section{Characterization of Transferosomal hydrogel}

\section{Vesicle Size and Entrapment Efficiency:}

Table includes the value of vesicle size, and entrapment efficiency. The vesicle size of all transfersomes varied between 178.89 and $245.65 \mathrm{~nm}$ whereas entrapment efficiency was found between 61.15 to $75.65 \%$.

Table.3. Vesicle size and Entrapment Efficiency

\begin{tabular}{cccc}
\hline S.No. & Formulation & $\begin{array}{c}\text { Vesicle Size } \\
(\mathrm{nm})\end{array}$ & $\begin{array}{c}\text { Entrapment } \\
\text { Efficient } \\
(\%)\end{array}$ \\
\hline 1 & F1 & $230.39 \pm 0.94$ & $61.98 \pm 0.62$ \\
3 & F2 & $211.71 \pm 0.25$ & $66.01 \pm 0.68$ \\
4 & F3 & $\mathbf{1 8 2 . 9 4 \pm 0 . 4 8}$ & $\mathbf{7 0 . 6 7 \pm 1 . 5 6}$ \\
5 & F4 & $196.29 \pm 0.20$ & $60.63 \pm 1.26$ \\
6 & F5 & $223.32 \pm 0.72$ & $63.13 \pm 1.49$ \\
\hline
\end{tabular}


Table 4: Vesicle size and entrapment efficiency of optimized formulation

\begin{tabular}{cccc}
$\begin{array}{c}\text { Formulation } \\
\text { Code }\end{array}$ & $\begin{array}{c}\text { Vesicle size } \\
(\mathrm{nm})\end{array}$ & $\begin{array}{c}\text { Entrapment } \\
\text { efficiency }\end{array}$ & $\begin{array}{c}\text { Zeta } \\
\text { potential } \\
(\mathrm{mv})\end{array}$ \\
\hline F3 & $182.94 \pm 0.48$ & $70.67 \pm 1.56$ & -25.1 \\
\hline
\end{tabular}

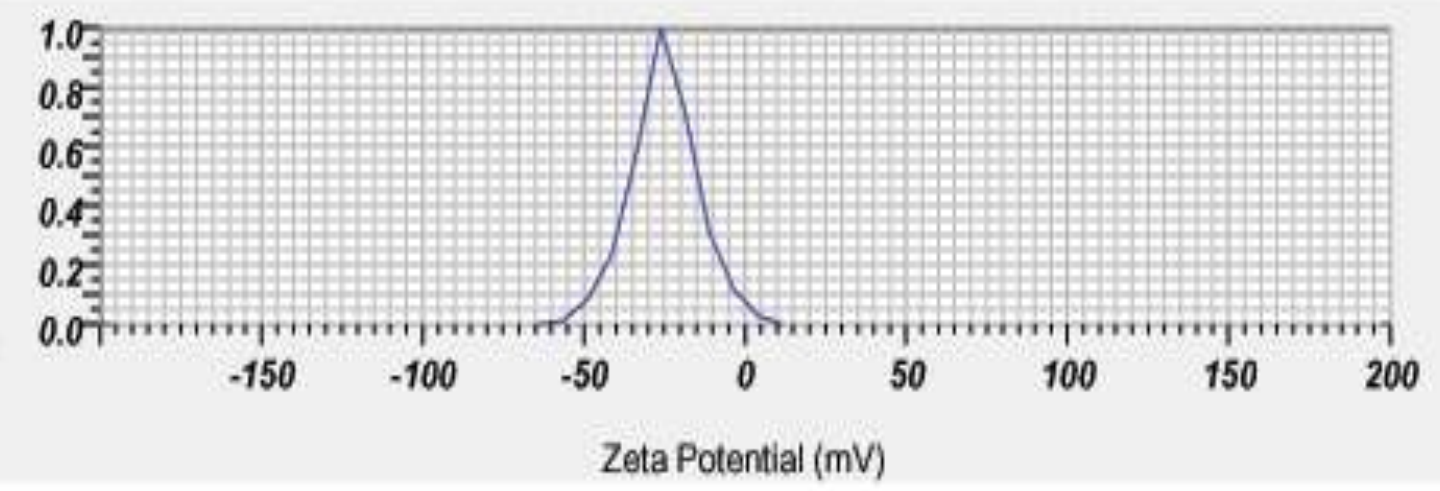

Figure 1. Zeta potential of optimize formulation

\section{Microscopic observation}

The photomicrographs showed the presence of spherically shaped vesicles dispersed in all the batches prepared.
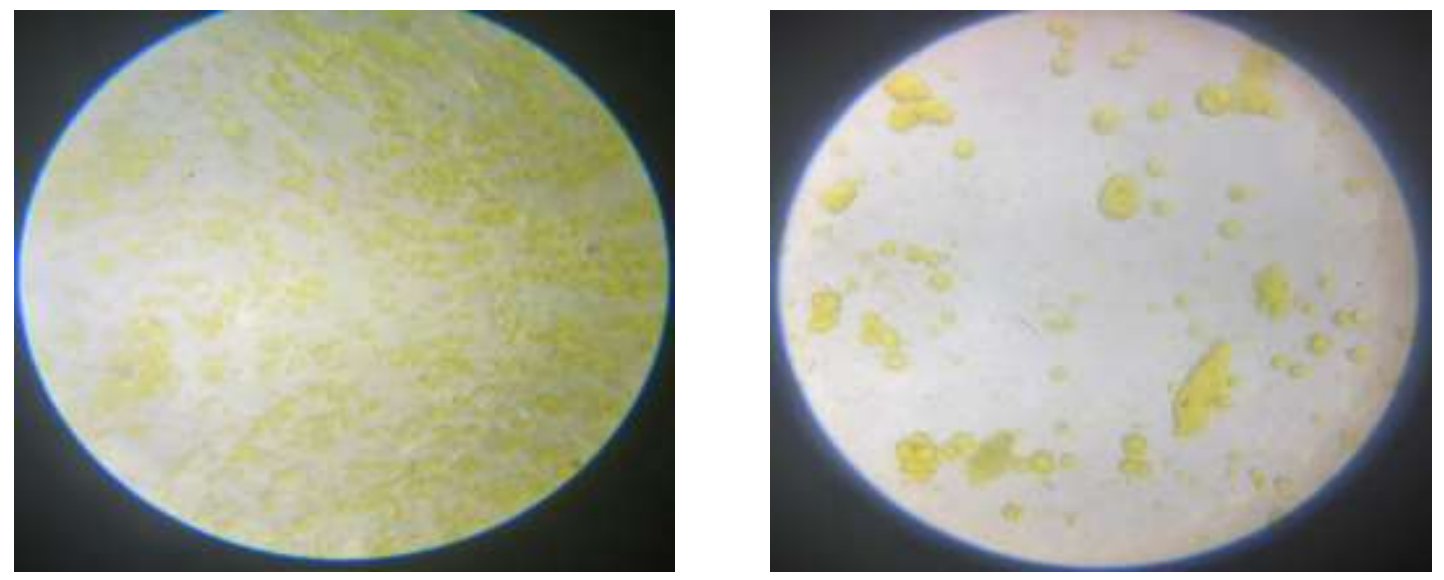

Figure 2: Microscopic observation of transfersomes of optimized formulations (F3) 


\section{Evaluation of Transferosomal Hydrogel:}

\section{Determination of pH}

The $\mathrm{pH}$ of the formulations was found to be satisfactory and was in the range of $6.7 \pm 0.038$ $6.9 \pm 0.039$, as shown in Table 5. The preparations were fluid at room temperature and at the $\mathrm{pH}$ formulated. Terminal sterilization via autoclaving had no impact on the $\mathrm{pH}$.

\section{Viscosity measurements}

The viscosity of gels was determined by using a Brookfield viscometer DV-II model. T-Bar spindle in combination with a helipath stand was used to measure the viscosity and have accurate readings.

The Viscosity of optimized formulation was found to be was in the range of $2361 \pm 0.046$ $2772 \pm 0.058$ cps.

\section{Spreadibility}

A modified apparatus was used for determining spreadability. The spreadability was measured on the basis of slip and drug characteristics of the gels and was in the range of $10.29 \pm 0.046-$ $11.57 \pm 0.053 \mathrm{gms}$. $\mathrm{cm}$. / $\mathrm{sec}$. The gels should have optimum spreadability because very high and very low spreadability values indicate that the application of the gel to the site is difficult. The spreadability of optimized formulation F-3 was found to be $11.57 \pm 0.053$.

\section{Drug content}

Drug content is most important in transfersomes formulation and the data found are satisfactory. It was found to be $96.61 \pm 0.028$ to $98.58 \pm 0.036 \%$ which shows the good capacity of formulation to hold the drug. The maximum drug content was found in formulation F-3 $(98.38 \pm 0.043 \%)$.

Table 5: Results of transfersomes hydrogel formulations

\begin{tabular}{cccccc}
\hline S.No. Formulation & pH & Viscosity & $\begin{array}{c}\text { Spreadability }(G \\
\text { m.cm/sec. }\end{array}$ & Drug content (\%) \\
\hline 1 & HG1 & $6.9 \pm 0.039$ & $2659 \pm 0.075$ & $11.08 \pm 0.042$ & $97.51 \pm 0.017$ \\
\hline & HG2 & $6.8 \pm 0.038$ & $2737 \pm 0.042$ & $10.75 \pm 0.059$ & $96.61 \pm 0.028$ \\
3 & HG3 & $\mathbf{6 . 9} \pm \mathbf{0 . 0 2 1}$ & $\mathbf{2 6 8 7} \pm \mathbf{0 . 0 5 9}$ & $\mathbf{1 1 . 5 7 \pm 0 . 0 5 3}$ & $\mathbf{9 8 . 3 8 \pm \mathbf { 0 . 0 4 3 }}$ \\
\hline & HG4 & $6.7 \pm 0.038$ & $2772 \pm 0.058$ & $10.83 \pm 0.058$ & $98.53 \pm 0.033$ \\
\hline
\end{tabular}




\section{In-vitro drug release study}

In-vitro diffusion study of the transfersomes hydrogel (F1-F6) was performed using modified Franz diffusion cell with dialysis membrane in phosphate buffer $\mathrm{pH} 7.4$ for a period of 12 hours. In vitro release of the drug was found in the rage of 80.269-89.230. (Table 6). In vitro release of the drug was fitted into different kinetic models like Zero-order, First-order, Higuchi model and Korsmeyer Peppas model. The zero-order plots were observed to be genuinely linear. In request to determine the precise system of medication discharge from curcumin gel the In-vitro discharge information was fitted to Korsmeyer Peppas was determined. As per the above research it was shown that the transferosome gel was discharged medication in controlled release way in 12 hour.

Table 6: In vitro release data of transferosomal using phosphate buffer 7.4

\begin{tabular}{cccccc}
\hline S. No & Time (hr) & HG1 & HG2 & HG3 & HG4 \\
\hline 1 & 0 & 0 & 0 & 0 & 0 \\
\hline 2 & 0.5 & 13.568 & 11.890 & 14.973 & 8.274 \\
3 & 1 & 22.336 & 20.210 & 19.709 & 14.115 \\
4 & 2 & 34.050 & 32.087 & 34.042 & 24.729 \\
5 & 4 & 48.638 & 44.733 & 49.684 & 41.461 \\
6 & 6 & 66.993 & 64.560 & 68.397 & 60.568 \\
7 & 8 & 78.074 & 74.046 & 77.553 & 70.648 \\
\hline 9 & 10 & 83.419 & 83.145 & 83.949 & 74.021 \\
\hline
\end{tabular}

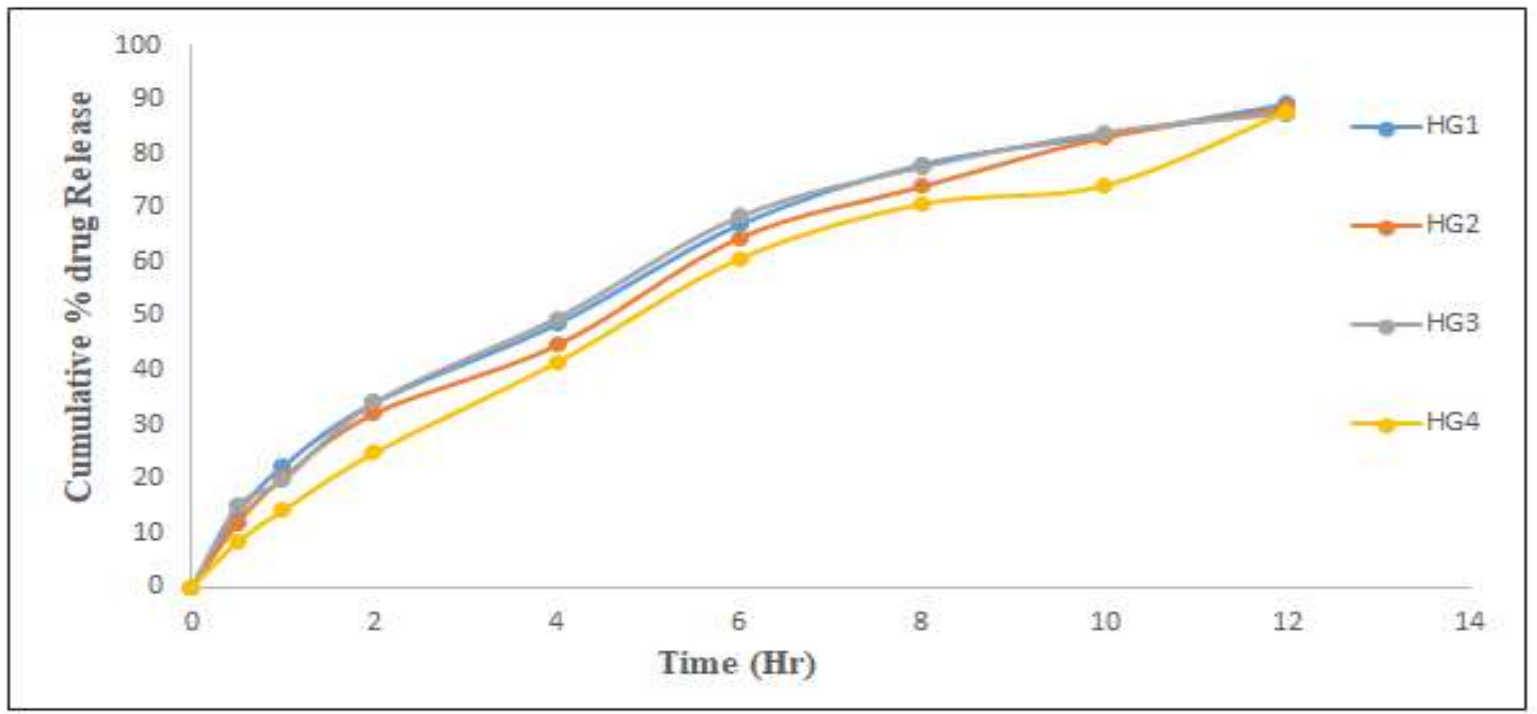




\section{CONCLUSION:}

From this study, it was concluded that the transferosomal formulations of curcumin, with high EE $\%$ and small particle size. Also, the preparation of curcumin as transfersomal gel has the ability to overcome the barrier properties of the skin and increase the drug release.

\section{REFERENCE:}

1. Ceve G. Transferosomes, liposomes and other lipid suspensions on the skin: permeation enhancement, vesicle penetration, and transdermal drug delivery. Crit. Rev. Ther. Drug Carrier Sysm. 1996; 257-388.

2. Ogihara U, Sasaki T, Toyama H, Sneha OM, Nishigori H. Rapid diagnostic imaging of cancer using radiolabeled liposomes. Cancer. Detect. Prev. 1997; 490-496.

3. Prajapati S. T, Patel C.G and Patel C.N, “ Transferosome: A vesicular carrier system for Transdermal drug delivery." Asian journal of Biochemical and Pharmaceutical Research, $2011 ; 1: 507-524$.

4. Brondsted H, Kopecek J, Harland RS, Prud'homme RK Eds. In Polyelectrolyte Gels. ACS Symposium Series 480. American Chemical Society. Washington, DC, 1992; Chapter 17.

5. Kunal Pal, Banthia AK and Majumdar DK. Starch based hydrogel with potential biomedical application as artificial skin. African Journal of Biomedical Research 2006; 9: 23-29.

6. Yasuda $\mathrm{K}$ et al., Biomechanical properties of high -toughness double network hydrogels. Biomaterials 2005; 26: 4468-4475.

7. Patel R, Singh SK, Singh S, Sheth NR, Gendle R. Development and Characterization of Curcumin Loaded Transfersome for Transdermal Delivery. J. Pharm. Sci. \& Res. 2009; 1(4): 71-80.

8. Jain CP, Vyas SP and Dixit VK. International Journal of Pharmaceutics, 2006; 68: 575-578.

9. Elsayed MMS, Abdallah OY and Nagar VF. International Journal of Pharmaceutics. 2006; 322: 60-66.

10. Duangjit S, Opanasopit P, Rojanarata T, Ngawhirunpat T. Evaluation of meloxicam-loaded cationic transfersomes as transdermal drug delivery carriers. AAPS PharmSciTech. 2013; 14:133-40.

11. Jaiswal P, Kesharwani S, Kesharwani R, Patel D, Ethosome: A new technology used as topical $\&$ transdermal delivery system. Journal of Drug Delivery and Therapeutics. 2016; 6(3):7-17. 
12. Modi CD and Bharadia PD, "Transfersomes: New Dominants for Transdermal Drug Delivery", American Journal of Pharmtech Research. 2012; 2(3):71-91.

13. Cevc G, Blume G, Scha“tzlein A. Transferosomes-mediated transepidermal delivery improves the regiospecificity and biological activity of corticosteroids in vivo. J Control Rel. 1997; 45: 211-26.

14. Jivrani Shilpa D and Patel Vijay K. Formulation, Development and Evaluation of Niosomal Drug Delivery System for Clindamycin Phosphate, Pharma Science Monitor. 2014; 5(2): 256274.

15. Mishra M and Biswal P. Complexation, Optimization, Formulation development and characterization of clindamycin phosphate gel using zinc acetate dehydrate, international jouranal of pharmacy, 2012; 2(3):472-486. 